\title{
Anti-HIV Therapy
}

National Cancer Institute

\section{Source}

National Cancer Institute. Anti-HIV Therapy. NCI Thesaurus. Code C16118.

Treatment of human immunodeficiency virus (HIV) infections with medications that target the virus directly, limiting the ability of infected cells to produce new HIV particles. 